\title{
Desempenho multicriterial no setor brasileiro de distribuição de energia elétrica: uma análise apoiada em DEA para os anos de 2007 e 2008
}

\author{
Marcelo Álvaro da Silva Macedo \\ Doutorado em Engenharia da Produção Pela Universidade Federal do Rio de Janeiro - UFRJ \\ Pós-Doutorado em Controladoria e Contabilidade pela FEA-USP \\ Professor do Departamento de Contabilidade da (FACC) - UFRJ \\ Av. Pasteur, 250 - Depto de Contabilidade/FACC/UFRJ, Urca, 22290-240, Seropedica, RJ \\ malvaro.facc.ufrj@gmail.com \\ Maitê Garcia Cruz Ribeiro \\ Mestranda em Ciências Contábeis da FACC - Universidade Federal do Rio de Janeiro - UFRJ \\ Av. Pasteur, 250 - Depto de Contabilidade/FACC/UFRJ, Urca, 22290-240, Seropedica, RJ \\ maitegcr@yahoo.com.br
}

Este artigo tem como objetivo analisar, por meio do uso da Análise Envoltória de Dados (DEA), o desempenho de empresas do setor brasileiro de distribuição de energia elétrica, tendo como base um conjunto de indicadores divididos em três dimensões/perspectivas: contábil-financeiro (modelo 01), operacional (modelo 02) e socioambiental (modelo 03). Todos os indicadores foram obtidos a partir da base de dados Séries, publicada pelo GESEL/NUCA/IE/UFRJ, para os anos de 2007 e 2008. Para análise foram utilizados modelos DEA-VRS, que foram implementados com o apoio do software SIAD, sendo considerados ajustes para valores negativos e restrições aos pesos. Os resultados mostram que as empresas que obtiveram melhores índices de eficiência nos três modelos foram: CPFL Mococa, Coelba, Elektro, CPFL Jaguari e Energipe. Já as que obtiveram os piores índices foram: Enersul, Celtins, Caiuá e EEB. Além disso, o teste de diferença de médias mostrou que não existe diferença entre os desempenhos médios dos grupos maiores e menores empresas e a análise de correlação mostrou que não existe correlação significativa entre desempenho e tamanho. Em outras palavras, pôde-se constatar que não é o tamanho, mas sim o desempenho superior, que confere competitividade às empresas.

Palavras-chave: Desempenho Multicriterial. DEA. Setor Elétrico.

\section{Multicriterial performance in Brazilian electricity distribution sector: an analysis} supported by DEA for the years 2007 and 2008

This article aims to analyze, through the use of DEA (Data Envelopment Analysis), the performance of the Brazilian eletricity distribution sector companies, based on a set of indexes apart in three 
Desempenho multicriterial no setor brasileiro de distribuição de energia elétrica: uma análise apoiada em DEA para os anos de 2007 e 2008

Marcelo Álvaro da Silva Macedo, Maitê Garcia Cruz Ribeiro

dimensions/perspectives: financial and accounting (model 01), operational (model 02) and socioenvironmental (model 03). All indicators were obtained from the database Series, published by GESEL/NUCA/IE/UFRJ, for the years 2007 and 2008. The analysis used the DEA-VRS, which were implemented with the support of SIAD software, being considered adjustments for negative values and weights restrictions. The results show that companies that had greater levels of efficiency in the three models were: CPFL Mococa, Coelba, Elektro, CPFL Jaguari e Energipe. On the other hand, those that had the worst rates were: Enersul, Celtins, Caiuá e EEB. Furthermore, the difference of means test showed no difference between the average performance of groups large and small companies, and correlation analysis showed no significant correlation between performance and size. In other words, we could see that it is not size, but superior performance, which gives the companies' competitiveness.

Keywords: Multicriterial Performance. DEA. Electricity Sector.

\section{INTRODUÇÃO}

O crescimento da produção interna dos países deve-se muito ao setor de infraestrutura, que possui impacto significativo sobre a produtividade geral da economia, o crescimento econômico e o bem-estar da população. Tal setor vem sendo caso de grande atenção nos últimos anos no Brasil, principalmente na área de Energia Elétrica devido, entre fatos como a sua expansão e modernização, à relevância de temas recentes tais como: aquecimento global, energia alternativa, eficiência energética, entre outros.

Atualmente, esse assunto se encontra em foco nas discussões e palestras de grandes empresas financiadoras, a exemplo do Sistema Eletrobrás e do Banco Nacional de Desenvolvimento Econômico e Social (BNDES), onde tem merecido atenção especial. Somente na área de energia estão previstos investimentos de $\mathrm{R} \$ 92$ bilhões de 2010 a 2013 , crescimento de 35,7\% em relação ao realizado de 2005-2008 (Portos e Navios, 2010).

Há, ainda, outro grande interesse tanto de empresas estrangeiras como de grandes organizações nacionais, devido à sistematização do problema energético global com a valorização e o desenvolvimento de tecnologia nas empresas de energia.

Por sua relevância econômica e social, o setor de energia elétrica no Brasil, assim como outros relacionados à infra-estrutura, tem suas atividades reguladas por agências ligadas ao Governo. No âmbito de energia, é a Agência Nacional de Energia Elétrica (ANEEL), vinculada ao Ministério das Minas e Energia (MME) que tem a função de regular as empresas que atuam no setor. As normas emanadas da ANEEL possuem diversas características e se

ABCustos Associação Brasileira de Custos - Vol. VI n², 78 - 100 - mai/ago 2011 
Desempenho multicriterial no setor brasileiro de distribuição de energia elétrica: uma análise apoiada em DEA para os anos de 2007 e 2008 Marcelo Álvaro da Silva Macedo, Maitê Garcia Cruz Ribeiro

referem a regras que vão desde a capacidade de geração de energia, passando por regulação tarifária até normas contábeis para divulgação de informações dessa natureza nos relatórios de tais empresas. Dessa forma, as empresas que atuam no setor elétrico estão sujeitas, além da legislação comercial e societária, às determinações da agência reguladora quanto à divulgação de informações financeiras.

Tendo em vista o histórico de crises e transformações nesse setor, além de mudanças constantes de sua regulamentação, é visto como necessários maiores estudos nessa área, sobretudo das práticas contábeis aplicadas. Por outro lado, os sistemas de medição do desempenho das empresas tornam-se fundamentais num mercado onde existe competitividade. A análise padronizada do desempenho de empresas que atuam nessa área, dada à consolidação em somente um indicador base para cada vertente, permite uma percepção mais clara do desenvolvimento de cada empresa frente à situação e direcionamento do setor, o que possibilita um laudo preciso para a tomada de decisão, tanto dos acionistas quanto dos investidores.

Desta forma, este trabalho teve como objetivo modelar o desempenho de 42 empresas distribuidoras de energia elétrica sob analise para os anos de 2007 e 2008, tendo mais de uma perspectiva/dimensão: contábil-financeira, operacional e socioambiental. Assim, busca-se verificar a sustentabilidade de cada empresa analisada, de forma comparativa e multicriterial, levando em consideração vetores/critérios de desempenho de naturezas distintas.

Neste contexto, o presente estudo apresenta o seguinte problema de pesquisa: qual o desempenho de cada empresa sob análise em cada um dos modelos (perspectivas) de desempenho?

Além disso, este trabalho se limita somente em considerar para fins de análise, o desempenho das empresas medido por três dimensões: socioambiental, operacional e contábil-financeira. Assim, considera-se que a sustentabilidade de um empreendimento será alcançada se a empresa obtiver eficiência em sua atuação nessas perspectivas. Isso não invalida os resultados do presente estudo, mas restringe seu foco.

\section{REFERENCIAL TEÓRICO}

\section{Setor Elétrico no Brasil}

No Brasil, o setor elétrico já passou por várias fases, tendo participação mais ativa do 
Desempenho multicriterial no setor brasileiro de distribuição de energia elétrica: uma análise apoiada em DEA para os anos de 2007 e 2008 Marcelo Álvaro da Silva Macedo, Maitê Garcia Cruz Ribeiro

setor privado, outras vezes do setor público e, em outras fases essa participação apresenta-se equilibrada entre os dois setores. A partir da década de 1960, o controle do setor elétrico foi assumido diretamente pelo setor público, centralizando sua política (ZIMMERMANN, 2007).

Até meados da década de 1990 o setor elétrico brasileiro era composto majoritariamente por empresas verticalmente integradas, com controle estatal nos níveis federal, estadual ou municipal. A partir de 1995, em decorrência de uma tendência internacional, teve início a implantação de uma reforma do modelo então vigente, com a aprovação da Lei №. 9.074. Com essa lei, foram dados os primeiros passos com a finalidade de introduzir a competição na geração e na comercialização de energia elétrica, bem como se iniciou o processo de privatização da distribuição (ZIMMERMANN, 2007).

No ano seguinte (1996) foi criada a Agência Nacional de Energia Elétrica - ANEEL pela Lei no 9.427, tendo entre seus objetivos: regular e fiscalizar a produção, transmissão e comercialização de energia elétrica (ANEEL, 1998). Em 1998, como resultado do Projeto de Reestruturação do Setor Elétrica (RE - SEB), a Lei no. 9.648 instituiu o Mercado Atacadista de Energia, e fez surgir um novo tipo de agente - os comercializadores. Além disso, esta lei estabeleceu o Operador Nacional do Sistema Elétrico - ONS.

Em 2002, o processo de reestruturação institucional do setor elétrico brasileiro, iniciada em 1995 ainda estava em andamento. Esta reestruturação previa a separação das atividades de geração, transmissão, distribuição e comercialização, reforçando a competitividade entre estas. Em 2001, foi interrompida por causa do "apagão", sendo estabelecida uma política de emergência, com consumo reprimido e grandes prejuízos gerados para os consumidores e para a economia nacional. Os investidores não estavam querendo investir mais nas hidrelétricas pelo elevado risco que estas ofereciam. Porém, estavam interessados muito mais nas usinas térmicas, pois o capital investido e o prazo de conclusão das obras são muito menores. E, apesar da energia gerada ter maiores custos, esses são suportados pelo consumidor.

Com relação à regulamentação do setor, o regime tarifário sofreu diversas mudanças. Começando pela remuneração garantida, a qual trazia um mecanismo de tarifas uniformes entre as concessionárias. Logo depois, com a preparação para a privatização, as concessionárias distribuidoras passaram a ter seus reajustes e tarifas fixadas de forma diferenciada, em função dos custos do serviço de cada uma (DIEESE, 2007). Conforme informações da ANEEL (2008), em 1997 houve um aumento elevado (aproximadamente 10\%) das tarifas. A partir de então, as tarifas passaram a ser definidas pelo novo agente regulador, 
Desempenho multicriterial no setor brasileiro de distribuição de energia elétrica: uma análise apoiada em DEA para os anos de 2007 e 2008 Marcelo Álvaro da Silva Macedo, Maitê Garcia Cruz Ribeiro

a ANEEL, criando um modelo para sua correção, denominado Price-Cap (fixa um valor máximo de tarifa e requer uma receita estimada por parte da empresa concessionária), tendo seu reajuste como base a variação do Índice de Preços ao Consumidor - Amplo (IPCA).

Após tantas transformações, o cenário atual encontra-se ainda em expansão e melhoramento, principalmente com a parte de regulamentação pelo Estado e oferta de energia pelas empresas, algumas privadas e outras ainda estatais. É, ainda, percebido o direcionamento de empresas para investimentos em energia limpa, realçando o novo cenário que o setor de energia vem buscando para o futuro.

\section{Desempenho organizacional}

A avaliação de desempenho é uma das ferramentas existentes para que se possa auxiliar no progresso do negócio (LAVIERI; CUNHA, 2009). Pois com o conhecimento da organização, maior o controle e a capacidade para melhorá-la.

O processo de mensurar o desempenho organizacional é um processo permanente e repetitivo. Através de tal avaliação, além do melhoramento de falhas, o gestor pode se preparar para enfrentar as mudanças ambientais a que a empresa está sujeita, sendo interessante para a companhia fazer tal mensuração em relação a seu ambiente competitivo (MACEDO et al., 2008).

Existem diversos estudos sobre qual a melhor forma de avaliar o desempenho organizacional de uma empresa. Uma gama de definições para desempenho organizacional foi criada, mas não há um consenso sobre qual definição seria a melhor ou uma receita específica de critérios e indicadores a serem utilizados, pois cada medida tem suas limitações e uma multiplicidade de abordagens que seria mais adequada (BARNEY, 1996).

De acordo com Machado, Machado e Holanda (2007), a mensuração do desempenho é um instrumento de gestão com o objetivo principal de proporcionar um gerenciamento eficaz da organização, sendo dependente de uma série de variáveis, como: bases informativas, variáveis consideradas, critérios, conceitos e princípios adotados.

Sendo assim, o desempenho organizacional ainda é um fenômeno que possui muitos aspectos, tornando-se muitas vezes complexo de se abordar. O que acaba por garantir uma maior definição ao tema, é a maneira que cada pesquisador o direciona e define os objetivos específicos de sua pesquisa. Ainda assim, o fato de organizar os distintos aspectos do desempenho em um todo integrado torna-se um estimável desafio (SILVA et.al., 2005). 
Desempenho multicriterial no setor brasileiro de distribuição de energia elétrica: uma análise apoiada em DEA para os anos de 2007 e 2008 Marcelo Álvaro da Silva Macedo, Maitê Garcia Cruz Ribeiro

Devido à complexidade do conceito de desempenho organizacional, muitos pesquisadores têm sugerido o uso de múltiplas medidas, pois, normalmente, não é possível delinear o sucesso de uma empresa fazendo uso puramente de uma única.

Por possuir diversos campos a serem analisados numa organização e pela contabilidade poder adentrar em seus diversos ramos, a definição e agrupamento de indicadores para cada dimensão é indispensável para que qualquer parecer seja elaborado. Esse processo demanda atenção e estudo minucioso do que é importante para o setor em que a empresa se insere e o que ela deve possuir de essencial para se manter competitiva no mercado.

Segundo Alves (2004), o modelo multidimensional para análise de organizações empresariais possibilita um estudo mais flexível e articulado, recusando a interpretação de fenômenos isolados e únicos. Ele incorpora, além das relações ambiente-empresa, a dinâmica de conjunto de indicadores e fatos opostos e ao mesmo tempo complementares.

Sendo assim, para a caracterização do modelo de avaliação de desempenho multidimensional, parte-se da s98(r)8.94808(t)-1.35th(-7) 
Desempenho multicriterial no setor brasileiro de distribuição de energia elétrica: uma análise apoiada em DEA para os anos de 2007 e 2008 Marcelo Álvaro da Silva Macedo, Maitê Garcia Cruz Ribeiro

de serem direcionadores para o alcance do desenvolvimento sustentável (GARCIA; GUERRERO, 2006). Além disso, incorporar a complexidade socioambiental no mundo dos negócios é fundamental. São valores predominantes na sociedade que devem ser estendidos às instituições.

A utilização dessas três perspectivas para a análise do desempenho organizacional, trás uma grande variedade de indicadores e métricas de eficiência, que de uma forma geral dificultam uma avaliação final, pois não fornecem aos tomadores de decisão uma medida única (MACEDO et al., 2008). Ou seja, complementam os autores, utilizar múltiplos critérios não necessariamente quer dizer gerar uma visão multicriterial. Para isso, é necessária a utilização de uma ferramenta de consolidação dos vetores de desempenho capaz de determinar uma métrica singular, representando a complexidade da análise, tal qual a Análise Envoltória de Dados (DEA).

Apesar de originalmente ser uma metodologia proposta em um ambiente de produção (transformação de insumos em produtos), salientam Macedo, Casa Nova e Almeida (2009), a DEA pode ser utilizada como um método multicritério, quando se utiliza indicadores do tipo quanto menor melhor no lugar dos inputs (ex.: risco, custo, endividamento, defeitos, reclamações etc.) e do tipo quanto maior melhor no lugar dos outputs (ex.: lucratividade, retorno, liquidez, benefícios socioambientais etc.). Isso transforma a DEA em um método de Apoio Multicritério à Decisão (AMD), aplicado com o intuito de consolidar várias perspectivas (critérios) de desempenhos diferentes.

A DEA já foi utilizada em vários trabalhos para consolidar medidas de desempenho organizacional, dentre os quais se destacam: Zhu (2000), Santos e Casa Nova (2005) e Macedo et al. (2008). É esta linha de pensamento que esta pesquisa segue, no que diz respeito à análise do desempenho organizacional.

\section{METODOLOGIA}

Conforme o exposto por Vergara (2004), o estudo realizado segue a natureza descritiva e quantitativa, pois se busca expor características a respeito do desempenho multidimensional das empresas distribuidoras de energia elétrica, que fazem parte da amostra, através da aplicação da DEA, combinado as perspectivas/dimensões contábil-financeira, operacional e socioambiental. 
Desempenho multicriterial no setor brasileiro de distribuição de energia elétrica: uma análise apoiada em DEA para os anos de 2007 e 2008 Marcelo Álvaro da Silva Macedo, Maitê Garcia Cruz Ribeiro

O processo de amostragem é não probabilístico, pois parte-se de um universo naturalmente restrito, pois as empresas do setor elétrico foram escolhidas pela disponibilidade e acessibilidade às informações necessárias ao estudo. Isso traz algumas limitações de inferência, mas não invalida os resultados da pesquisa, já que não se tem por objetivo generalizar os achados.

Esta pesquisa foi feita a partir de dados secundários colhidos na edição de 2009 do Anuário Séries 2009, editado pela Eletrobrás e produzido em conjunto com o Grupo de Estudos do Setor Elétrico - GESEL/NUCA/IE/UFRJ. As organizações escolhidas têm como premissa inicial serem empresas Distribuidoras do Setor de Energia Elétrica.

Inicialmente, havia 42 empresas distribuidoras listadas no anuário, entretanto, devido à inconsistência como, por exemplo, Patrimônio Líquido negativo, e/ou insuficiência de informações apresentadas por algumas das empresas, a amostra desse estudo se reduziu a 35 empresas.

De cada uma das empresas selecionadas foram coletadas informações/critérios, que foram divididas em três modelos de análise do desempenho organizacional, cada um com uma perspectiva/dimensão diferente: contábil-financeira, operacional e socioambiental.

A escolha destas variáveis/critérios se deve ao fato destes serem, além dos principais grupos disponíveis na publicação utilizada, os mais relevantes, considerados por diversos autores no estudo do setor elétrico, e que conseguem representar a atuação nestas diversas dimensões. A seguir tem-se uma descrição sucinta de cada índice/critério em cada modelo utilizado:

- Modelo 01: Desempenho Contábil-Financeiro - Procura-se verificar a eficiência de cada empresa em termos de vetores contábil-financeiros relacionados à questão do endividamento, dos investimentos em ativos permanentes, da necessidade capital de giro e da rentabilidade.

- Endividamento Oneroso (EnO): Representa o input 01 e é obtido pela divisão dos Empréstimos, Financiamentos e Debêntures (Curto e Longo Prazo) pelo Patrimônio Líquido (exceto a Reserva de Reavaliação). Mede o percentual de capital próprio comprometido com as dívidas de curto e longo prazo. Mostra, portanto, o risco financeiro, sendo, assim, um indicador do tipo quanto menor melhor.

- Imobilização dos Recursos não correntes (IRNC): Representa o input 02 e é calculado a partir da divisão do Ativo Imobilizado pelo somatório do Patrimônio Líquido (exceto a 
Desempenho multicriterial no setor brasileiro de distribuição de energia elétrica: uma análise apoiada em DEA para os anos de 2007 e 2008

Marcelo Álvaro da Silva Macedo, Maitê Garcia Cruz Ribeiro

Reserva de Reavaliação) e do Passivo Não Circulante. Indica que percentual de Recursos não correntes a empresa aplicou no Ativo Permanente. É um indicador do tipo quanto menor melhor.

- Necessidade de Investimento em capital de giro sobre o Ativo Total (NIG/AT): Representa o input 03 e é obtido pela divisão do Passivo Circulante Operacional, subtraído do Ativo Circulante Operacional, pelo Ativo Total. Demonstra a parcela que a necessidade de investimento em giro da empresa representa do Patrimônio total da empresa. Como o NIG é a parte dos investimentos operacionais não financiada pelos respectivos passivos, em questão à quota do Ativo, é considerado um indicador do tipo quanto menor, melhor o desempenho.

- Retorno sobre o PL (RSPL): Representa o output 01 e mede a remuneração (em termos de lucro) dos acionistas obtida pela empresa em relação ao investimento próprio realizado. É calculado partir da divisão do Lucro do período pelo Patrimônio Líquido. É um indicador do tipo, quanto maior, melhor o desempenho.

- Retorno sobre o Ativo (RSA): Representa o output 02 e é calculado partir da divisão do Lucro do Período Antes do Imposto de Renda e Contribuição Social, pelo Ativo Operacional. É um indicador do tipo, quanto maior melhor o desempenho, pois indica quanto a empresa gera de lucro em relação ao capital investido.

- Modelo 02: Desempenho Operacional - Procura-se verificar a eficiência da empresa no atendimento aos seus clientes e na transformação de receita em lucro. A idéia é verificar o desempenho tendo como input a qualidade dos serviços prestados e como output a margem de lucro operacional. A eficiência é alcançada pelas empresas que possuem alta qualidade de serviços prestados (baixos DEC e FEC), alta margem de lucro operacional (EBITDA) e alto fluxo de caixa operacional.

- DEC: Representa o input 01 e mede a duração de interrupção equivalente por consumidor, medida pelo número de horas que, em média, cada consumidor ficou desligado no período. Mede, portanto, a qualidade da prestação do serviço de distribuição, sendo um índice do tipo quanto menor, melhor o desempenho.

- FEC: Representa o input 02 e mede o número de interrupções ocorridas, em média, em cada unidade consumidora. Também mede, sob outra perspectiva, a qualidade do serviço prestado, sendo um índice do tipo quanto menor melhor o desempenho. 
Desempenho multicriterial no setor brasileiro de distribuição de energia elétrica: uma análise apoiada em DEA para os anos de 2007 e 2008

Marcelo Álvaro da Silva Macedo, Maitê Garcia Cruz Ribeiro

- Margem EBIT (MEbit): Representa o output 01, sendo a divisão do EBIT (Earnings Before Interest and Taxes) pela Receita Líquida. Mede, portanto, o percentual de lucro que a empresa conseguiu obter em sua operação (descontadas as receitas e despesas financeiras). É um indicador do tipo quanto maior, melhor o desempenho.

- Fluxo de Caixa Operacional sobre a Receita Líquida (FCO/RL): Representa o output 02. É a divisão do Fluxo de Caixa Operacional pela Receita Líquida. Mede, portanto, o percentual de caixa que a empresa conseguiu obter em sua operação, sendo um indicador do tipo quanto maior, melhor o desempenho.

- Modelo 03: Desempenho Sócio-Ambiental - Procura-se verificar qual a eficiência de cada empresa na conversão da capacidade de investimento (input), medida pela receita líquida, em benefícios sócio-ambientais (outputs), medidos pelos indicadores sociais internos e externos e pelos indicadores ambientais. A lógica do modelo é observar a relação entre capacidade de investimento e benefícios socioambientais, de forma que quanto maiores forem os benefícios para uma menor capacidade de investimento, maior será a eficiência e, por conseguinte, o desempenho sócio-ambiental da empresa.

- Indicador Interno sobre Receita Líquida (II/RL): Representa o input 01 e mede o total de recursos investidos no bem-estar dos funcionários em relação à Receita Líquida. É um índice do tipo quanto maior, melhor o desempenho, portanto, na análise é um indicador calculado como complementar a 100\%.

- Indicador Externo sobre Receita Líquida (IE/RL): Indicador que representa o input 02, sendo obtido pela soma de todos os recursos investidos pelas empresas em benefício da sociedade, em áreas tais como: educação, saúde, cultura etc; em relação à Receita Líquida. É um índice do tipo quanto maior, melhor o desempenho, portanto, na análise é um indicador calculado como complementar a $100 \%$.

- Investimento em Meio Ambiente sobre Receita Líquida: Representa o input 03 e mede os investimentos na área ambiental em relação à Receita Líquida. Por conta disso, é um índice do tipo quanto maior, melhor o desempenho, portanto, na análise é um indicador calculado como complementar a $100 \%$.

- Valor Adicionado a Distribuir sobre Receita Líquida (VAD/RL): Representa o output 01. Mede o quanto de riqueza líquida a empresa tem a distribuir em relação à Receita Líquida. Sendo assim, é um índice do tipo quanto maior, melhor o desempenho. 
Desempenho multicriterial no setor brasileiro de distribuição de energia elétrica: uma análise apoiada em DEA para os anos de 2007 e 2008 Marcelo Álvaro da Silva Macedo, Maitê Garcia Cruz Ribeiro

Após a definição dos indicadores, foi feita uma escolha do critério de seleção dos valores dos dados das empresas por meio de cálculo de Quartil. Calculou-se o 1ำ e o $3^{\circ}$ Quartil, e em seguida foi realizada a análise de outliers por meio do intervalo interquartílico. Assim sendo, mesmo que a DEA ressalte a importância dos outliers, decidiu-se excluir os valores extremos, eliminando aqueles distantes três vezes o intervalo interquartílico do quartil superior e do quartil inferior.

Das 35 empresas analisadas, cinco empresas apresentaram percentuais muito acima ou muito abaixo do estimado como, por exemplo, o indicador de Retorno sobre o Patrimônio Líquido, a CPFL Paulista apresentou um indicador de 945,3\%, enquanto que o calculo da máxima estipulada para esse indicador resultou em 126\%. Dessa maneira, a amostra se reduziu a 30 empresas.

O que se busca neste artigo é apresentar e discutir uma metodologia, na qual seja possível avaliar o desempenho de cada empresa do ramo de distribuição de energia elétrica de modo multicriterial, ou seja, considerando de maneira integrada todos os vetores de desempenho apresentados em cada modelo. Além disso, busca-se uma visão ao longo do tempo, para evitar oscilações esporádicas e pontuais e por fim uma visão integrada/multidimensional dos três modelos analisados.

Isto é feito através da utilização da Análise Envoltória de Dados (DEA), que de acordo com Macedo, Casa Nova e Almeida. (2009), mostra-se como uma metodologia de avaliação de desempenho capaz de comparar a eficiência de várias unidades operacionais homogêneas (Decision Making Units - DMUs) mediante a exposição do uso de suas múltiplas entradas (inputs) para a produção de múltiplas saídas (outputs). Ou seja, apresenta a eficiência de uma unidade/empresa em relação às outras, em consideração a seus inputs e outputs.

Nesse caso, para utilização do modelo é necessário identificar as DMU's, as entradas (inputs) e as saídas (outputs). As DMU's são as unidades operacionais similares da avaliação, que neste estudo são as empresas distribuidoras de energia elétrica. Para a utilização do modelo é de fundamental importância que as DMU's sejam homogêneas. Os outputs referemse aos resultados obtidos pelas DMU's e compõem o numerador do quociente de eficiência. Estes devem respeitar o critério matemático de quanto maior, melhor. Os inputs são os recursos utilizados pelas DMU's para obter os outputs e devem obedecer ao critério de quanto menor, melhor, compondo o denominador do quociente de eficiência (KASSAI, 2002).

São várias as formulações dos modelos de DEA encontradas na literatura, conforme mencionam Macedo et. al. (2008), entretanto dois modelos básicos DEA são geralmente 
Desempenho multicriterial no setor brasileiro de distribuição de energia elétrica: uma análise apoiada em DEA para os anos de 2007 e 2008 Marcelo Álvaro da Silva Macedo, Maitê Garcia Cruz Ribeiro

usados nas aplicações. O primeiro modelo chamado de CCR (CHARNES et al., 1978), também conhecido como CRS (Constant Returns to Scale), avalia a eficiência total, identifica as DMU's eficientes e ineficientes e determina a que distância da fronteira de eficiência estão às unidades ineficientes. O segundo chamado de modelo BCC (BANKER; CHARNES; COOPER, 1984), também conhecido como VRS (Variable Returns to Scale), utiliza uma formulação que permite a projeção de cada DMU ineficiente sobre a superfície de fronteira (envoltória) determinada pelas DMU's eficientes de tamanho compatível. Assim sendo, o modelo DEA-VRS é mais aplicável quando não se tem homogeneidade de tamanho entre as unidades sob análise.

Segundo Macedo et. al. (2008), um caminho intuitivo para introduzir DEA é por meio de forma de razão. Para cada unidade (DMU), procura-se de obter uma medida de razão de todos os outputs sobre todos os inputs, ou seja, os pesos ótimos $u_{j}$ e $v_{i}$ são obtidos pela resolução do seguinte problema de programação matemática:

$$
\begin{aligned}
\text { Max } E_{c}= & \frac{\sum_{j=1}^{s} u_{j} y_{j c}}{\sum_{i=1}^{m} v_{i} x_{i c}} \\
\text { S.a.: } \quad & \frac{\sum_{j=1}^{s} u_{j} y_{j k}}{\sum_{i=1}^{m} v_{i} x_{i k}} \leq 1, k=1,2, \ldots, c, \ldots, n \\
& u_{j} \geq 0, \forall j, \\
& v_{i} \geq 0, \forall i
\end{aligned}
$$

Neste modelo, ressalta Macedo, Casa Nova e Almeida (2009), c é a unidade (DMU) que está sendo avaliada. O problema acima envolve a procura de valores para u e v, que são os pesos, de modo que maximize a soma ponderada dos outputs (output "virtual") dividida pela soma ponderada dos inputs (input "virtual") da DMU em estudo, sujeita a restrição de que esse quociente seja menor ou igual a um, para todas as DMU's. Esta função está sujeita à restrição de que, quando o mesmo conjunto de coeficientes de entrada e saída (os vários $v_{i}$ e $u_{j}$ ) for aplicado a todas as outras unidades de serviços que estão sendo comparadas, nenhuma unidade excederá $100 \%$ de eficiência ou uma razão de 1,00.

Ainda de acordo com os autores, este é um problema fracionário (não linear) de programação matemática que pode ser mais facilmente resolvido transformando a relação em 
Desempenho multicriterial no setor brasileiro de distribuição de energia elétrica: uma análise apoiada em DEA para os anos de 2007 e 2008 Marcelo Álvaro da Silva Macedo, Maitê Garcia Cruz Ribeiro

uma função linear, simplesmente considerando o denominador (soma ponderada dos insumos) da função objetivo igual a um. Os modelos DEA-CRS e DEA-VRS podem então ser apresentados da seguinte maneira:

$$
\begin{aligned}
& \operatorname{Max} E_{c}=\sum_{j=1}^{s} u_{j} y_{j c} \\
& \operatorname{Max} E_{c}=\sum_{j=1}^{s} u_{j} y_{j c}+u^{\prime} \\
& \text { S.a.: } \quad \sum_{i=1}^{m} v_{i} x_{i c}=1 \\
& \text { S.a.: } \quad \sum_{i=1}^{m} v_{i} x_{i c}=1 \\
& \sum_{j=1}^{s} u_{j} y_{j k}-\sum_{i=1}^{m} v_{i} x_{i k} \leq 0, k=1,2, \ldots, c, \ldots, n \\
& \sum_{j=1}^{s} u_{j} y_{j k}-\sum_{i=1}^{m} v_{i} x_{i k}+u^{\prime} \leq 0, k=1,2, \ldots, c, \ldots, n \\
& u_{j}, v_{i} \geq 0, \forall i, j \text {. } \\
& u_{j,} v_{i} \geq 0, \forall i, j \text {. }
\end{aligned}
$$

$\mathrm{Na}$ análise conduzida neste artigo, um modelo VRS é utilizado, já que as unidades sob análise possuem tamanhos diferentes. No modelo DEA-VRS, c é a unidade (DMU - Decision Making Units) que está sendo avaliada e u' é uma variável irrestrita, que responde pela questão dos retornos variáveis de escala. O problema acima envolve a procura de valores para $u_{j}$ e $v_{i}$, que são os pesos, de modo que maximize a soma ponderada dos outputs $\left(y_{j}\right) d a$ DMU em estudo, sujeita as restrições de que a soma ponderada dos inputs $\left(\mathrm{x}_{\mathrm{i}}\right)$, desta mesma DMU, seja igual a um e a diferença entre a soma ponderada dos outputs $\left(y_{j}\right)$ e a soma ponderada dos inputs $\left(\mathrm{x}_{\mathrm{i}}\right)$ seja menor ou igual a zero para todas as DMU's. Esta última restrição faz com que quando o mesmo conjunto de coeficientes de entrada e saída (os vários $\mathrm{u}_{\mathrm{j}}$ e $\mathrm{v}_{\mathrm{i}}$ ) for aplicado a todas as outras unidades que estão sendo comparadas, nenhuma unidade excederá $100 \%$ de eficiência.

Tendo o banco de dados para a análise, alguns ajustes foram necessários tendo em vista que valores negativos não seriam aceitos. Par 
Desempenho multicriterial no setor brasileiro de distribuição de energia elétrica: uma análise apoiada em DEA para os anos de 2007 e 2008 Marcelo Álvaro da Silva Macedo, Maitê Garcia Cruz Ribeiro

obtenção da eficiência de cada DMU utilizou-se um software de DEA, apresentado por Meza et al.. (2003), denominado SIAD (Sistema Integrado de Apoio à Decisão). Depois para cada perspectiva obteve-se um único fator de desempenho composto pela média dos valores de cada ano (2007 e 2008). Depois com os valores de cada perspectiva obteve-se uma nova média para calcular o desempenho global de cada empresa no período de análise.

Um ponto importante que deve ser ressaltado é que em cada perspectiva utilizou-se o artifício matemático de manter pesos iguais para cada variável do modelo. Assim, em cada modelo cada variável tem peso igual e no final a consolidação feita também considera que cada perspectiva também tem importância similar. Esta é uma importante limitação do presente estudo, pois com pesos diferentes poder-se-ia obter desempenhos diferentes. Apesar de ser uma limitação, esta estrutura de pesos pelo menos garante a não eliminação de qualquer variável no modelo de análise, já que a modelagem DEA poderia atribuir peso zero às variáveis de cada unidade que tivessem comportamento prejudicial ao cálculo de seu desempenho. Com este artifício garante-se a utilização de todas as dimensões do desempenho na computo do indicador final.

Com o intuito de fazer algumas análises comparativas entre as empresas da amostra, aplicaram-se alguns testes de diferenças de média, a partir dos indicadores de desempenho obtidos pela DEA em cada perspectiva de análise em cada ano. Ou seja, para se proceder a comparação entre os índices de grupos de empresas, utilizou-se de testes de diferença de médias ao nível de significância de $5 \%$.

Para verificar se as unidades maiores tinham desempenho superior às unidades de menor porte, utilizou-se o teste não-paramétrico para diferença de médias de Mann-Whitney, que, segundo Siegel e Castellan Jr. (2006), é uma boa alternativa ao teste paramétrico $t$, 
Desempenho multicriterial no setor brasileiro de distribuição de energia elétrica: uma análise apoiada em DEA para os anos de 2007 e 2008 Marcelo Álvaro da Silva Macedo, Maitê Garcia Cruz Ribeiro

\section{ANÁLISE DOS RESULTADOS}

Para analisar as informações das empresas distribuidoras de energia elétrica, montouse um modelo de avaliação de eficiência para cada perspectiva/dimensão em cada ano (2007 e 2008).

Conforme pode se verificar, a tabela 01 mostra a eficiência de cada empresa em cada modelo a cada ano (Eff 2007 e Eff 2008). Posteriormente, foi calculada a média dos anos (Me Eff). Nesse sentido, apresentou-se um único desempenho para cada empresa e para cada modelo.

Para o Modelo 01 observa-se que em 2007 seis empresas (AES Sul, Cosern, CPFL Jaguari, CPFL Mococa, Elektro e Energipe) obtiveram índice máximo de desempenho. Já no ano de 2008, o número de empresas subiu para oito, incluindo-se as CEEE D, Coelba, CPFL Leste Paulista e Copel D, sendo que somente a AES Sul e a Elektro não conseguiram manter tal índice no ano seguinte. Consolidando então os resultados dos dois anos para o Modelo 1, obtiveram índices máximos de eficiência as empresas: Cosern, CPFL Jaguari, CPFL Mococa e Energipe. Já as empresas Ampla, Caiuá, Celtins, EDEVP e Enersul, tiveram as piores eficiências para o ano de 2007. Com relação ao ano de 2008, Caiuá, Celtins e EDVP tiveram uma piora ainda maior em sua eficiência.

No Modelo 02, pode-se verificar o máximo de eficiência para o ano de 2007 de quatro empresas (CFLO, CPFL Mococa, Elektro e Energipe). Aumentando esse quadro para cinco no ano de 2008 com a inclusão de três empresas (Coelba, CPFL Jaguari e Energisa Borborema) e perda de eficiência de duas empresas, Elektro e Energipe. Com o cálculo da média dos anos, somente duas empresas se mantiveram no topo na questão de eficiência: CFLO e CPFL Jaguari.

Entretanto, nesse mesmo modelo, verifica-se a baixa eficiência no ano de 2007 de onze empresas (AES Sul, Ampla, Caiuá, CEEE D, Celtins, CNEE, Copel D, EEB, Enersul, Energisa Borborema e Energisa NF). Para o ano de 2008, todas as empresas obtiveram melhora nesse índice. Mesmo assim, continuaram a possuir baixa eficiência as empresas AES Sul, CEEE D, Celtins, CNEE, EEB e Energisa NF.

Tabela 01 - Resultado do Desempenho de cada Empresa a cada ano em cada perspectiva

\begin{tabular}{|c|c|c|c|c|c|c|c|c|c|}
\hline \multirow[b]{2}{*}{ Empresa } & \multicolumn{3}{|c|}{ Modelo 1} & \multicolumn{3}{|c|}{ Modelo 2} & \multicolumn{3}{|c|}{ Modelo 3} \\
\hline & $\begin{array}{c}\text { Eff } \\
2007\end{array}$ & $\begin{array}{c}\text { Eff } \\
2008\end{array}$ & Me Eff & $\begin{array}{c}\text { Eff } \\
2007\end{array}$ & $\begin{array}{c}\text { Eff } \\
2008\end{array}$ & Me Eff & $\begin{array}{c}\text { Eff } \\
2007\end{array}$ & $\begin{array}{c}\text { Eff } \\
2008\end{array}$ & Me Eff \\
\hline
\end{tabular}

ABCustos Associação Brasileira de Custos - Vol. VI n², 78 - 100 - mai/ago 2011 
Desempenho multicriterial no setor brasileiro de distribuição de energia elétrica: uma análise apoiada em DEA para os anos de 2007 e 2008 Marcelo Álvaro da Silva Macedo, Maitê Garcia Cruz Ribeiro

\begin{tabular}{|c|c|c|c|c|c|c|c|c|c|}
\hline AES SUL & 1,0000 & 0,5729 & 0,7864 & 0,3966 & 0,4536 & 0,4251 & 0,8767 & 0,8872 & 0,8820 \\
\hline AMPLA & 0,4192 & 0,4346 & 0,4269 & 0,3429 & 0,6246 & 0,4838 & 0,9274 & 0,9111 & 0,9192 \\
\hline BANDEIRANTE & 0,6244 & 0,5896 & 0,6070 & 0,6886 & 0,6986 & 0,6936 & 0,8991 & 0,8934 & 0,8962 \\
\hline CAIUÁ & 0,3414 & 0,2096 & 0,2755 & 0,4175 & 0,6439 & 0,5307 & 0,9170 & 0,9239 & 0,9205 \\
\hline CEMIG & 0,5567 & 0,5014 & 0,5291 & 0,6279 & 0,6905 & 0,6592 & 1,0000 & 1,0000 & 1,0000 \\
\hline COSERN & 1,0000 & 1,0000 & 1,0000 & 0,7187 & 0,8512 & 0,7850 & 0,8903 & 0,9096 & 0,9000 \\
\hline CEEE D & 0,5417 & 1,0000 & 0,7708 & 0,1471 & 0,2167 & 0,1819 & 0,9111 & 0,9194 & 0,9153 \\
\hline COELBA & 0,7776 & 1,0000 & 0,8888 & 0,9289 & 1,0000 & 0,9645 & 0,9569 & 0,9282 & 0,9425 \\
\hline CELTINS & 0,4191 & 0,3624 & 0,3908 & 0,3383 & 0,4271 & 0,3827 & 0,9755 & 0,9564 & 0,9660 \\
\hline CELPE & 0,6196 & 0,7952 & 0,7074 & 0,5816 & 0,7632 & 0,6724 & 0,9241 & 520 & 0,9380 \\
\hline COELCE & 085 & 0,6 & 0,5608 & 0,6440 & 0,8583 & 12 & 41 & 37 & 0,9089 \\
\hline CFLO & 0,5821 & 0,5 & 0,5644 & 1,0000 & 1,0000 & 1,0000 & 366 & 546 & 0,9606 \\
\hline CPFL Jaguari & 1,0000 & 1,0000 & 1,0000 & 0,8004 & 1,0000 & 0,9002 & 0,8718 & 367 & 0,8543 \\
\hline CPFL Mococa & 1,0000 & 1,0000 & 1,0000 & 1,0000 & 1,0000 & 1,0000 & 0,9274 & 0,9005 & 0,9139 \\
\hline CNEE & 372 & 457 & & 0,4132 & 0,4517 & 0,4325 & 0,9247 & 0,9201 & 0,9224 \\
\hline \multicolumn{10}{|l|}{ CPFL Leste } \\
\hline Paul & 825 & 1,0000 & 0,7913 & 0,7008 & 0,7997 & 0,7502 & 0,9207 & 0,8868 & 0,9038 \\
\hline \multicolumn{10}{|l|}{ CPFL Sul } \\
\hline Paulista & 0,7389 & 0,9000 & 0,8194 & 0,6755 & 0,9455 & $0, \varepsilon$ & 0,9100 & 0,9092 & 0,9096 \\
\hline Copel D & 0,6902 & 1,0000 & 0,8451 & 0,3539 & 0,5779 & 0,4659 & 0,9909 & 1,0000 & 0,9954 \\
\hline Elektro & 1,0000 & 0,7032 & 0,8516 & 1,0000 & 0,9994 & 997 & 2225 & 192 & 0,9208 \\
\hline Eletropaulo & 0,6257 & 0,9495 & 0,7876 & 0,7228 & 0,8685 & 0,7957 & 241 & 0,8999 & 0,9120 \\
\hline EDEVP & 0,4238 & 0,3935 & 0,4087 & 0,6050 & 0,7419 & 0,6734 & 0,9199 & 0,9201 & 0,9200 \\
\hline EEB & 0,4712 & 0,4214 & 0,4463 & 0,1696 & 0,2376 & 0,2036 & 0,9123 & 0,9210 & 0,9167 \\
\hline Enersul & 0,3243 & 0,4542 & 0,3893 & 0,2037 & 0,6714 & 0,4376 & 0,9444 & 0,8911 & 0,9178 \\
\hline Energipe & 1,0000 & 1,0000 & 1,0000 & 1,0000 & 0,6717 & 0,8359 & 0,9032 & 0,9130 & 0,9081 \\
\hline \multicolumn{10}{|l|}{ Energisa } \\
\hline Borborema & 0,6843 & 0,9473 & 0,8158 & 0,3572 & 1,0000 & 0,6786 & 0,8763 & 0,9101 & 0,8932 \\
\hline Energisa MG & 0,8178 & 0,9137 & 0,8657 & 0,7364 & 0,6077 & 0,6721 & 0,9125 & 0,8895 & 0,9010 \\
\hline Energisa NF & 0,5326 & 0,5407 & 0,5367 & 0,4129 & 0,4768 & 0,4449 & 0,9057 & 0,9152 & 0,9104 \\
\hline Energisa PB & 0,6161 & 0,7090 & 0,6626 & 0,5310 & 0,5223 & 0,5267 & 0,9348 & 0,9080 & 0,9214 \\
\hline Escelsa & 0,5123 & 0,4456 & 0,4790 & 0,5636 & 0,7657 & 0,6646 & 0,9819 & 0,9204 & 0,9511 \\
\hline RGE & 0,4978 & 0,4971 & 0,4974 & 0,4231 & 0,5205 & 0,4718 & 0,9049 & 0,8958 & 0,9004 \\
\hline
\end{tabular}

Já, ao ser analisado o Modelo 03, apenas uma empresa (Cemig) conseguiu alcançar a máxima eficiência para o ano de 2007. A Cemig conseguiu manter seu nível de eficiência também para o ano de 2008, tendo como companhia em tal fator a Copel D. Contudo, nenhuma empresa obteve baixa eficiência nesse índice. Sendo a média mais baixa dos anos resultando em 0,8543 da CPFL Jaguari.

Com base nos indicadores de desempenho de cada modelo em cada ano procedeu-se uma análise para verificar se existia relação entre tamanho (AT) e desempenho. A primeira análise que foi conduzida foi uma comparação entre as médias dos grupos de empresas (maiores e menores). As médias para cada modelo em cada ano para cada um destes grupos 
Desempenho multicriterial no setor brasileiro de distribuição de energia elétrica: uma análise apoiada em DEA para os anos de 2007 e 2008 Marcelo Álvaro da Silva Macedo, Maitê Garcia Cruz Ribeiro

é mostrada na tabela 02.

Tabela 02 - Eficiência Média para cada Modelo dos Grupos Maiores e Menores Empresas

\begin{tabular}{|c|c|c|c|c|c|c|}
\hline \multirow{2}{*}{ Grupos } & \multicolumn{3}{|c|}{2007} & \multicolumn{3}{c|}{2008} \\
\cline { 2 - 7 } & Eff Mod 01 & Eff Mod 02 & Eff Mod 03 & Eff Mod 01 & Eff Mod 02 & Eff Mod 03 \\
\hline Maiores Empresas & 0,6320 & 0,6314 & 0,9364 & 0,7084 & 0,7602 & 0,9303 \\
\hline Menores Empresas & 0,6653 & 0,6135 & 0,9135 & 0,7688 & 0,8023 & 0,9043 \\
\hline
\end{tabular}

Os resultados dos testes não paramétricos de diferença de médias de Mann-Whitney, para um nível de significância de 5\%, mostram que para todos os casos as médias dos grupos maiores e menores é igual. Ou seja, os $p$-values encontrados para os seis testes mostram que não se pode rejeitar a $\mathrm{H} 0$ de que as médias dos grupos sejam iguais ao nível de $5 \%$.

Além disso, uma análise complementar mostra que nenhum dos desempenhos, de nenhum modelo em nenhum ano, está correlacionado com o tamanho. $\mathrm{Na}$ verdade, as correlações de Spearman calculadas não são significativas ao nível de $5 \%$, ou seja, não há elementos para rejeitar a $\mathrm{H} 0$ de que estas correlações são iguais a zero.

Em síntese, o teste de diferença de médias mostrou que não existe diferença entre os desempenhos médios dos grupos maiores e menores empresas e a análise de correlação mostrou que não existe correlação significativa entre desempenho e tamanho. Em outras palavras, pôde-se constatar que não é o tamanho, mas sim o desempenho superior que confere competitividade às empresas.

Após a análise de eficiência das empresas distribuidoras para os três modelos e para os dois anos em questão, foi elaborada a Tabela 03 que une os desempenhos de cada modelo em um desempenho geral.

Deste modo, encontram-se ranqueadas as empresas em ordem de eficiência geral, ou seja, considerando a eficiência consolidada dos três modelos. As empresas que obtiveram melhores índices de eficiência nos três modelos foram: CPFL Mococa, Coelba, Elektro, CPFL Jaguari e Energipe. Já as que obtiveram os piores índices foram: Enersul, Celtins, Caiuá e EEB.

Considerando o Modelo 3 (socioambiental), percebeu-se que todas as trinta empresas obtiveram altos índices de eficiência, pois ao considerar o índice medido de 0 (zero) a 1 (um), todas as empresas tiveram índices superiores a 0,5 (mediana). Já nos Modelos 1 (contábil financeiro) e 2 (operacional), apenas vinte e duas e vinte, respectivamente, superaram a mediana. Tal fato pode ser verificado pela alta demanda de investimentos em meio ambiente 
Desempenho multicriterial no setor brasileiro de distribuição de energia elétrica: uma análise apoiada em DEA para os anos de 2007 e 2008 Marcelo Álvaro da Silva Macedo, Maitê Garcia Cruz Ribeiro

que as empresas vêm realizando ao decorrer dos anos.

Tabela 03 - Ranking Final de Desempenho

\begin{tabular}{|c|l|c|c|c|r|}
\hline Ranking & \multicolumn{1}{|c|}{ Empresa } & Ef Modelo 1 & Eff Modelo 2 & Eff Modelo 3 & Eff Geral \\
\hline 1 & CPFL Mococa & 1,0000 & 1,0000 & 0,9139 & 0,9713 \\
\hline 2 & COELBA & 0,8888 & 0,9645 & 0,9425 & 0,9319 \\
\hline 3 & Elektro & 0,8516 & 0,9997 & 0,9208 & 0,9240 \\
\hline 4 & CPFL Jaguari & 1,0000 & 0,9002 & 0,8543 & 0,9182 \\
\hline 5 & Energipe & 1,0000 & 0,8359 & 0,9081 & 0,9147 \\
\hline 6 & COSERN & 1,0000 & 0,7850 & 0,9000 & 0,8950 \\
\hline 7 & CPFL Sul Paulista & 0,8194 & 0,8105 & 0,9096 & 0,8465 \\
\hline 8 & CFLO & 0,5644 & 1,0000 & 0,9606 & 0,8417 \\
\hline 9 & Eletropaulo & 0,7876 & 0,7957 & 0,9120 & 0,8318 \\
\hline 10 & CPFL Leste Paul. & 0,7913 & 0,7502 & 0,9038 & 0,8151 \\
\hline 11 & Energisa MG & 0,8657 & 0,6721 & 0,9010 & 0,8129 \\
\hline 12 & Energisa Borborema & 0,8158 & 0,6786 & 0,8932 & 0,7959 \\
\hline 13 & CELPE & 0,7074 & 0,6724 & 0,9380 & 0,7726 \\
\hline 14 & Copel D & 0,8451 & 0,4659 & 0,9954 & 0,7688 \\
\hline 15 & COELCE & 0,5608 & 0,7512 & 0,9089 & 0,7403 \\
\hline 16 & BANDEIRANTE & 0,6070 & 0,6936 & 0,8962 & 0,7323 \\
\hline 17 & CEMIG & 0,5291 & 0,6592 & 1,0000 & 0,7294 \\
\hline 18 & Energisa PB & 0,6626 & 0,5267 & 0,9214 & 0,7035 \\
\hline 19 & Escelsa & 0,4790 & 0,6646 & 0,9511 & 0,6982 \\
\hline 20 & AES SUL & 0,7864 & 0,4251 & 0,8820 & 0,6978 \\
\hline 21 & EDEVP & 0,4087 & 0,6734 & 0,9200 & 0,6674 \\
\hline 22 & CNEE & 0,5415 & 0,4325 & 0,9224 & 0,6321 \\
\hline 23 & Energisa NF & 0,5367 & 0,4449 & 0,9104 & 0,6307 \\
\hline 24 & RGE & 0,4974 & 0,4718 & 0,9004 & 0,6232 \\
\hline 25 & CEEE D & 0,7708 & 0,1819 & 0,9153 & 0,6227 \\
\hline 26 & AMPLA & 0,4269 & 0,4838 & 0,9192 & 0,6100 \\
\hline 27 & Enersul & 0,3893 & 0,4376 & 0,9178 & 0,5815 \\
\hline 28 & CELTINS & 0,3908 & 0,3827 & 0,9660 & 0,5798 \\
\hline 29 & CAIUÁ & 0,2755 & 0,5307 & 0,9205 & 0,5756 \\
\hline 30 & EEB & 0,4463 & 0,2036 & 0,9167 & 0,5222 \\
\hline & & & & \\
\hline
\end{tabular}

Por fim, foi elaborado um gráfico de dispersão que representa a consolidação dos resultados encontrados na análise multidimensional pela média dos dois anos, em forma gráfica, que se encontra a seguir (Figura 01). A idéia neste ponto da análise é consolidar todas as perspectivas/dimensões, de maneira visual, de modo a propiciar uma análise conjunta dos índices utilizados na análise multicriterial através da DEA.

Nesta figura o eixo $Y$ representa o Modelo 01 e o eixo $X$ o Modelo 02. Logo, em cada figura tem-se quatro quadrantes possíveis formados pelas combinações alto e baixo desempenhos em cada modelo. Estabeleceu-se que o ponto mediano da escala seria o ponto de corte para a classificação em alto e baixo desempenho. Assim sendo, indicadores superiores a 0,5 são considerados bons e abaixo são ruins. Para incorporar o Modelo 03, seria utilizado o artifício de dividir cada quadrante em duas regiões: a superior onde ter-se-ia 
Desempenho multicriterial no setor brasileiro de distribuição de energia elétrica: uma análise apoiada em DEA para os anos de 2007 e 2008 Marcelo Álvaro da Silva Macedo, Maitê Garcia Cruz Ribeiro

empresas com desempenho acima de 0,5 no Modelo 03 e a inferior com empresas com baixo desempenho neste.

Porém, é notado na Tabela 03 que todas as empresas obtiveram índices superiores a $50 \%$ no Modelo 03, sendo assim desnecessária a consolidação deste modelo no gráfico já que todas as empresas apresentaram muito boa eficiência.

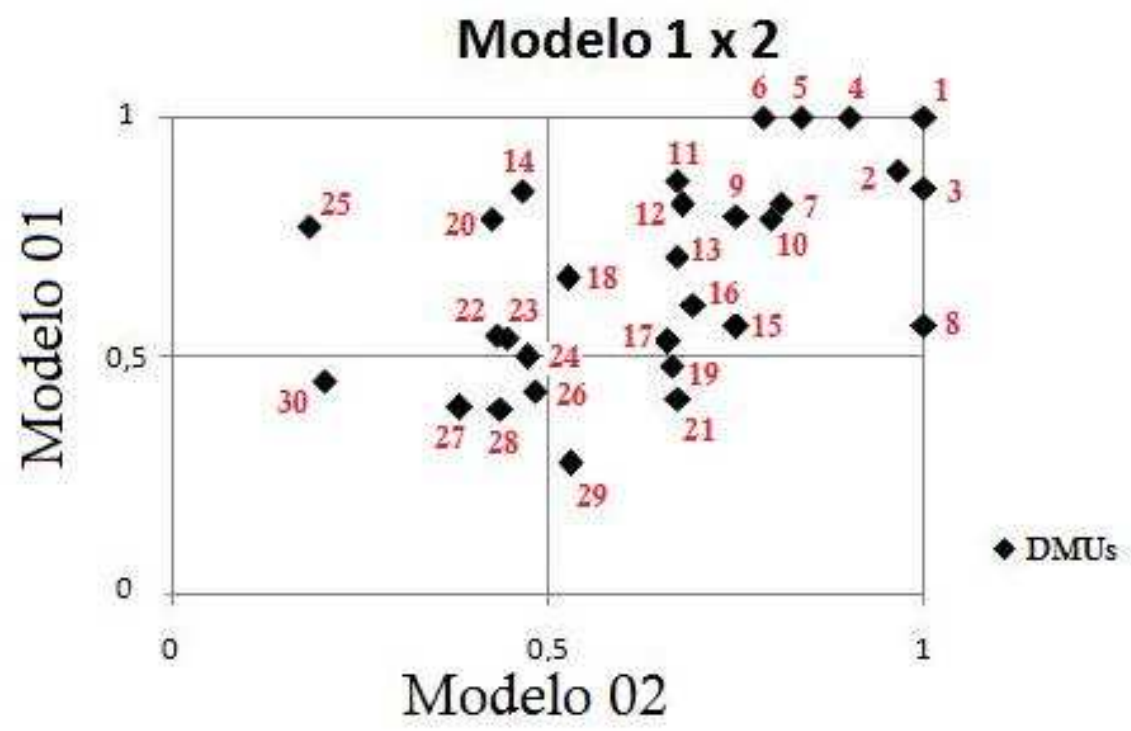

Figura 01 - Análise Multidimensional

Nesta análise, verifica-se que cinco empresas (RGE, Ampla, Enersul, Celtins e EEB) se encontram no quadrante que considera os piores índices para os Modelos 01 e 01 . Oito empresas estão alocadas nos quadrantes que possuem ou piores índices para o Modelo $01 \mathrm{e}$ melhores para o Modelo 02 (Copel D, AES Sul, CNEE, Energisa NF e CEEE D), ou o contrário, piores índices para o Modelo 02 e melhores índices para o Modelo 01 (Escelsa, EDEVP e Caiuá). Por fim, dezessete empresas se alocaram no quadrante que possui os melhores índices para os dois Modelos.

\section{CONSIDERAÇÕES FINAIS E CONCLUSÃO}

Procurou-se analisar, neste trabalho, o desempenho multidimensional de empresas brasileiras de distribuição de energia elétrica, nos anos de 2007 e 2008, por meio da Análise 
Desempenho multicriterial no setor brasileiro de distribuição de energia elétrica: uma análise apoiada em DEA para os anos de 2007 e 2008 Marcelo Álvaro da Silva Macedo, Maitê Garcia Cruz Ribeiro

Envoltória de Dados, a partir da análise multicriterial da atuação das mesmas nas perspectivas contábil-financeira, operacional e socioambiental.

A amostra utilizada no estudo foi de 30 (trinta) empresas, sendo utilizados para análise os modelos DEA-VRS, que foram implementados com o apoio do software SIAD, sendo considerados ajustes para valores negativos e restrições aos pesos.

Os resultados mostram que em relação à dimensão contábil-financeira apenas a Cosern, CPFL Jaguari, CPFL Mococa e Energipe, atingiram o índice de desempenho máximo (100\%) nos dois anos, enquanto que a Caiuá apresentou os piores resultados. Já para a dimensão operacional apenas a CFLO e a CPFL Mococa atingiram esse índice. Em contrapartida, a CEEE D se mostrou com os piores resultados nessa perspectiva. Nessa mesma dimensão, dez empresas apresentaram índices inferiores a 50\%, o que indica que a questão operacional nas empresas de energia deve ser atentada.

No modelo socioambiental, somente a Cemig alcançou tal índice para os dois anos. Ainda em relação ao modelo 03 (socioambiental) percebe-se que nenhuma das empresas em nenhum dos anos tem desempenho inferior a $50 \%$. Ou seja, as empresas deste setor têm desempenho socioambiental bom e pouco disperso, onde as melhores empresas não se distanciam tanto assim das piores.

Em relação à análise multidimensional, percebe-se que as empresas CPFL Mococa, Coelba, Elektro, CPFL Jaguari e Energipe são as com melhor desempenho no período, enquanto que a EEB se revela como a pior empresa.

Os resultados desse estudo propõem uma nova percepção sobre o desempenho de empresas, se referindo a atuação contábil-financeira, operacional e socioambiental, que não 
Desempenho multicriterial no setor brasileiro de distribuição de energia elétrica: uma análise apoiada em DEA para os anos de 2007 e 2008 Marcelo Álvaro da Silva Macedo, Maitê Garcia Cruz Ribeiro

AGENCIA NACIONAL DE ENERGIA ELÉTRICA (ANEEL). 2008. Legislação $30 / 01 / 2010$.

- Disponível em http://www.aneel.gov.br, acesso em

ALVES, S. A

Revista de Administração Contemporânea, v. 8, n. 2, 2004, pp. 71-93.

BANKER, R. D.; CHARNES, A.; COOPER, W. W. Some Models for Estimating Technical and Scale Inefficiencies in Data Envelopment Analysis. .v. 30, n. 9, 1984, p.1078-1092.

BARNEY, Jay B.

Company, 1996.

. MA: Addison-Wesley Publishing

CHARNES, A.; COOPER, W. W.; RHODES, E.

European Journal of Operational Research. v. 2, n. 6, 1978, 429-444.

DIEESE. 2008.

Nota Técnica no 58, Dez. 2007. Disponível em: http://www. dieese.org.br/notatecnica/notatec58Tarifa Energia.pdf. Acesso em: 30 jun. 2010.

FONTES, S. V.; MACEDO, M. A. S.

Nacional da ANPAD 2003, Atibaia.

Atibaia: ANPAD, $1 \mathrm{CD}$.

In: XXVII Encontro

GARCIA, S.; GUERRERO, M.

n. 35, 2006, p.45-57.

. Rev. Geogr. Norte Gd.,

KASSAI, S.

. São Paulo, SP. Tese de doutorado em Contabilidade e Controladoria. Faculdade de Economia, Administração e Contabilidade, Universidade de São Paulo, 2002, 318 p.

LAVIERI, C. A. E CARNEIRO DA CUNHA, J. A. $2009,1 \mathrm{CD}$.

. In: XXXIII Encontro Nacional da ANPAD, Anais... São Paulo: ANPAD,

MACEDO, M. A. S. ; CASA NOVA, S. P. C.; ALMEIDA, K. de

. Contabilidade, Gestão e Governança, v. 12, n. 3, 2009, p. 87-101.

MACEDO, M. A. S.; CÍPOlA, F. C.; FERReirA, A. F. R.; SOUZA, M. F. A. de.

2008, Rio de Janeiro. Rio de Janeiro: ANPAD, $1 \mathrm{CD}$.

In: XXXII Encontro Nacional da ANPAD,

MACHADO, M.; MACHADO, M. A.; HOLANDA, F. M. da.

. Turismo - Visão e Ação, v. 9, n. 3, 2007, p. 393-406.

MATARAZZO, D. C.

. 6. ed. São Paulo: Atlas, 2007, 459 p.

MeZA, L. A.; BiOndi Neto, L; SOARes De Mello, J. C. C. B.; GOMES. E. G. ; COELhO, P. H. G.. 
Desempenho multicriterial no setor brasileiro de distribuição de energia elétrica: uma análise apoiada em DEA para os anos de 2007 e 2008 Marcelo Álvaro da Silva Macedo, Maitê Garcia Cruz Ribeiro

MARINHA, 6, 2003, Rio de Janeiro. Anais... Rio de Janeiro: CASNAV, 1 CD.

In: SIMPÓSIO DE PESQUISA OPERACIONAL DA PORTOS E NAVIOS.

. Noticiário cotidiano - Geral. 22 de Fevereiro de 2010. Disponível em: http://www.portosenavios.com.br/site/noticiario/geral/1299-bndes-preve-alta-de-37-noinvestimento-em-4-setores-de-infraestrutura-ate-2013. Acesso em: 05 ago.2010.

SANTOS, A.; CASA NOVA, S. P. C. 2005.

. RAE-eletrônica. v. 4, n. 1, art. 8. Disponível em: http://www.rae.com.br/eletronica. Acesso em: 13 jan. 2008.

SIEGEL, S. ; CASTELLAN Jr., N. J. .2 ed. Porto Alegre: Artmed, 2006.

SILVA, J. F. ; CARNEIRO, J. M. T. ; ROCHA, A.; HEMAIS, C. A.

Bertrand. (Org.). Estudos em Negócios IV. Rio de Janeiro: Mauad, 2005.

In: Margarida Gutierrez; Hélène VERGARA, S. C. 2004, $112 \mathrm{p}$.

5. ed. São Paulo: Atlas,

ZHU, J. Multi

. European Journal of Operational Research. v. 123, n. 1, 2000, p. 105-124.

ZIMMERMANN, M. P.

Rio de Janeiro, RJ. Dissertação de mestrado em Engenharia Elétrica. Pontifícia Universidade Católica do Rio de Janeiro, 2007, 312p.

Submissão: 29/11/2010

Aceite: $27 / 07 / 2011$ 